\title{
Distribution of multiple sclerosis lesions detected by brain magnetic resonance imaging in Erbil city
}

\begin{abstract}
Background and objective: Many epidemiological studies and clinical manifestation studies of multiple sclerosis have been done in Iraq. Up to our knowledge, no such observational study to the radiological feature of the multiple sclerosis lesion has been done yet in Erbil in comparison to other worldwide studies. This study aimed to assess the distribution of multiple sclerosis lesions in brain regions detected by magnetic resonance imaging among Erbil population.

Methods: This was a cross-sectional study conducted at the College of Medicine, Hawler Medical University, from April 2018 to July 2019. A review of magnetic resonance imaging scans of the brain of 120 patients was done. Special attention was directed toward identifying the variance in multiple sclerosis lesions distribution in the brain regions and their MR signal intensity characteristics.

Results: Periventricular lesions were observed in more than $90 \%$ of the study sample. The next common was juxtacortical lesions (24.8\%), followed by corpus callosum lesions $(16.8 \%)$, while brain stem lesions were the least observed proportions. No significant difference was detected in the distribution of multiple sclerosis lesions among ethnicities and genders, except for basal ganglia lesions, which were significantly more common in women $(P=0.016)$. The magnetic resonance imaging signal intensity of the lesion was significantly variable among disease duration.
\end{abstract}

Conclusion: The T2 hyper intense lesions were most commonly seen in the periventricular region. Juxtacortical and corpus callosum lesions were also frequently observed. The proportions of the brain stem and cerebellum lesions appeared to be lower in comparison to previous studies.

Keywords: Multiple Sclerosis; Magnetic Resonance Imaging; Distribution; Lesion.

\section{Introduction}

Multiple sclerosis (MS) is the most common chronic inflammatory demyelinating disease of the central nervous system that affects adults. ${ }^{1}$ The lesions of multiple sclerosis basically appear within the optic nerves, spinal cord, brainstem, and the periventricular white matter of the cerebral hemispheres. ${ }^{2}$ Many studies stated that MS lesion is an area of hyperintensity on a T2 weighted MRI scan that is at least $3 \mathrm{~mm}$ on the long axis. ${ }^{3,4}$ Patients during the disease course might show an average of 5-10 new lesions and one or two clinical attacks annually. ${ }^{5}$ These active lesions are strongly correlated with relapse of clinical symptoms in MS patients but become less frequent during progressive MS cases. ${ }^{6}$ Conventional Magnetic resonance imaging (MRI) is a reliable non-invasive technique for MS diagnosis and has high sensitivity, revealing more than $90 \%$ of macroscopic tissue changes. ${ }^{2}$ According to MacDonald Criteria 2017, MS diagnosis requires an objective method to diagnose and monitor disease activity over time in symptomatic or asymptomatic patients. As a result, MRI is a reliable window to detect lesions in at least two of four locations (juxtacortical, periventricular, infratentorial, and spinal-

${ }^{1}$ Department of Surgery, College of Medicine, Hawler Medical University, Erbil, I raq.

* Correspondence: dtawfeeq84@gmail.com 
cord) (dissemination in space) with the integration of new lesions on follow up scans (dissemination in time) ${ }^{7,8}$ MS was thought previously as a white matter (WM) disease only. However, the involvement of grey matter in the pathological studies of brain samples of MS patients during autopsy has revealed the distribution of cortical pathology. ${ }^{9}$ Several studies have shown that MS can be presented as a focal lesion, normal appearing white matter, gray matter involvement with brain atrophy, and spinal lesions. Their distribution varies between Asian and Western populations. ${ }^{2,7}$, In Asian patients, studies revealed less cerebral hemisphere involvement. In fact, the optic-spinal type of MS is more frequent. $^{10}$ While in the Gulf states, MS plaques were found in the optic nerve, cerebral hemispheres, brain stem, and cerebellum. ${ }^{11}$ In Iraq, the most presented lesion is optic neuritis, followed by brain stem lesion about $25 \%$ and cerebellar involvement ranging from $7-11 \% .{ }^{12,13}$ Many epidemiological studies and clinical manifestation studies of MS have been done in Iraq. ${ }^{1,4,15}$ Up to our knowledge, no such observational study to the radiological feature of the MS lesion has been done yet in Erbil compared to other worldwide studies. This study aimed to assess the distribution of MS lesions in the brain regions detected by $\mathrm{MRI}$ among Erbil population and their relation to gender and to evaluate the MS lesions characteristic like size, signal intensity, and contrast enhancement, and their association with the duration or different disease stages (first appearing cases, chronic cases with clinical attacks, and chronic cases).

\section{Methods}

This observational cross-sectional study was conducted from April 2018 to July 2019. The study protocol was approved by the research ethics committee of the College of Medicine, Hawler Medical University. Written informed consent was obtained from each participant before any study procedure was carried out. Clinical brain MRI sequences for 120 adult patients with MS disease attending MRI units in the Erbil Teaching Hospitals with clinical criteria of MS or for follow-up scanning. Each patient was examined for the distribution and morphology of brain lesions. Data collection was carried out for seven months. We prospectively recruited MS patients who fulfilled the following inclusion criteria: diagnosed patients according to the revised McDonald's diagnostic criteria 2017, adult patient age ranging from 20 to 60 years, ability to walk with or without support or restricted to bed or chair or pushed in a wheelchair, regardless disability status scale score. The exclusion criteria were previous cerebrovascular diseases like transient ischemic attack or stroke, positive history of vascular risk factor such as, diabetes, hypertension and hyperlipidemia, systemic lupus erythematosus (SLE), and phospholipid syndrome, the presence of acute disseminated encephalomyelitis and neuromyelitis optica (NMO), and positive history of migraine. The brain MRI findings were interpreted by two specialist radiologists with experience of more than ten years in neuroradiology and were reviewed in the workstation, and some cases were on CDs based on radiological and clinical findings. Additional information was collected through interviewing the patients in the radiology department prior to or post examination. The patients with MS were scanned by 1.5 Tesla super-conducting magnets (Siemens MRI Machine). The standardized MRI protocol included axial and sagittal planes of T1 Weighted Images (T1WI), T2 Weighted Images (T2WI), and fluid-attenuated inversion recovery (FLAIR) sequences of the brain, axial Diffusion-weighted imaging (DWI) and/or apparent diffusion coefficient (ADC) sequences with b-value of 1000 . Additional images we obtained using axial and sagittal T1WI sequences after contrast administration to patients. 
The contrast was gadolinium (Gd) with diethylenetriamine pentacetate (DTPA) $(0.5 \mathrm{mmol} / \mathrm{ml})=(287 \mathrm{mg} / \mathrm{l})$ in dose of $0.2 \mathrm{ml} / \mathrm{kg}$ IV. For detailed quantitative analyses, the number of T2 hyper intense MS plaques $\geq 3 \mathrm{~mm}$ was visually counted, and the maximum diameter (millimeter) was measured. We follow the McDonald criteria for Diagnosis of MS 2017 in studying the lesion location. Therefore; The identified locations of the MS lesions in the white matter were categorized regions into periventricular white matter, juxtacortical white matter, corpus callosum cerebellum, brain stem (midbrain, pons, and medulla oblongata), and basal ganglia. Periventricular lesions are oval or elliptic hyperintense lesions on T2weighted images that are perpendicular to the anteroposterior axis of the head) (Dawson finger lesions) and perpendicular to the lateral ventricle and also including the lesions in the centrum semiovale. ${ }^{16}$ While isolated juxtacortical lesions are the plaques found along the subcortical U-fibers, and corpus callosum lesions are those found within corpus callosum oriented perpendicular to the ependyma. ${ }^{16}$ The rest of the lesions are referred to according to each site found within. The inftratentorial lesions are those lesions found in the brain stem and cerebellum. ${ }^{17}$ Moreover, additional lesion characteristics were analyzed. Tumefactive MS lesions are lesions more than $20 \mathrm{~mm}$, causing mass effect and edema, and/or has ring enhancement). ${ }^{18} \mathrm{~T} 1$ black hole lesions are of low signal intensity compared with the white matter on T1-weighted images and are concordant with hyperintense lesions on T2-weighted images. ${ }^{19}$ Active MS lesions are encountered in patients who did not have them in their previous MRI brain scan. Normal appearing white matter is when the characteristic aspects of MS pathology are not confined in the brain. ${ }^{20}$ A comparison was also made among three subgroups according to the disease's duration into new, acute on chronic, and chronic. New cases are the patients that are recently diagnosed by a committee of neurologists based on clinical, radiological manifestations with the integration of CSF analysis and Lab tests. Acute on chronic are the cases that are already diagnosed but presented with new active lesions and new objective neurological signs lasting for at least 24 hours. Chronic cases are those already diagnosed and clinically stable with no relapse of symptoms. The statistical package for the social sciences (version 22) was used for data analysis. Chi-square test analysis was used to compare the proportions, and Fisher's exact test was used when the expected count of more than $20 \%$ of the cell is less than 5. The student $t$ test for two independent samples was used to compare the means of two groups (e.g., males and females). A $P$ value of less than 0.05 was considered statistically significant.

\section{Results}

Out of 120 patients, 15 cases were excluded from the study because they had one of the exclusion criteria. The study sample comprises 105 patients, ranging from 18 to 60 years, with a mean age $\pm S D$ of $37.6 \pm 10.7$ years. As seen in Table 1, the most common age group (36\%) was between 36 - 45 years. 73 patients $(69.5 \%)$ were females and 32 patients $(30.5 \%)$ were males. The new cases were 11 patients (10.5\%), and chronic cases were the highest with $60 \%$ in proportion. Periventricular lesions were observed in more than $90 \%$ of the study sample. Regarding the number of lesions in each site, the most common location for lesion distribution was found in the deep white matter of cerebral hemispheres and mostly in periventricular region (36.6\%), as shown in Table 2. Frequent locations of MS lesions were also seen in juxtacortical area $(24.8 \%)$ and corpus callosum lesions $(16.8 \%)$. Around $23.8 \%$ of patients had brain stem lesions, including the midbrain, pons, and medulla oblongata, which were the least common affected sites compared 
to other locations (Figure 1).

Table 1: Basic characteristics of the study sample.

\begin{tabular}{lcc}
\hline Variables & No. & (\%) \\
\hline Age group in years & & \\
under 25 & 21 & $(20.0)$ \\
$26-35$ & 23 & $(21.9)$ \\
$36-45$ & 36 & $(34.3)$ \\
$46-55$ & 20 & $(19.0)$ \\
56 and older & 5 & $(4.8)$ \\
Gender & & \\
Male & 32 & $(30.5)$ \\
Female & 73 & $(69.5)$ \\
Ethnicity & & \\
Kurdish & 92 & $(87.6)$ \\
Arabic & 13 & $(12.4)$ \\
Duration & & \\
New & 11 & $(10.5)$ \\
Acute on chronic & 31 & $(29.5)$ \\
Chronic & 63 & $(60.0)$ \\
Total & 105 & $(100.0)$ \\
\hline
\end{tabular}

Table 2: Distribution of Brain MRI lesion among the population of the study sample.

\begin{tabular}{lccc}
\hline Variables of lesion site & \multicolumn{2}{c}{ Lesions } & $\begin{array}{c}\text { Percentage of patients affected } \\
\text { (N=105) }\end{array}$ \\
\hline Periventricular region & 100 & $(36.5)$ & 95.2 \\
Juxtacortical region & 68 & $(24.8)$ & 64.8 \\
Corpus callosum & 49 & $(17.9)$ & 46.7 \\
Cerebellum & 20 & $(7.3)$ & 19 \\
Basal ganglia & 12 & $(4.4)$ & 11.4 \\
Mid brain (cerebral peduncles) & 10 & $(3.6)$ & 9.5 \\
Pons & 13 & $(4.7)$ & 12.4 \\
Medulla oblongata & 2 & $(0.7)$ & 1.9 \\
Total & $\mathbf{2 7 4}$ & $(100.0)$ & \\
\hline
\end{tabular}

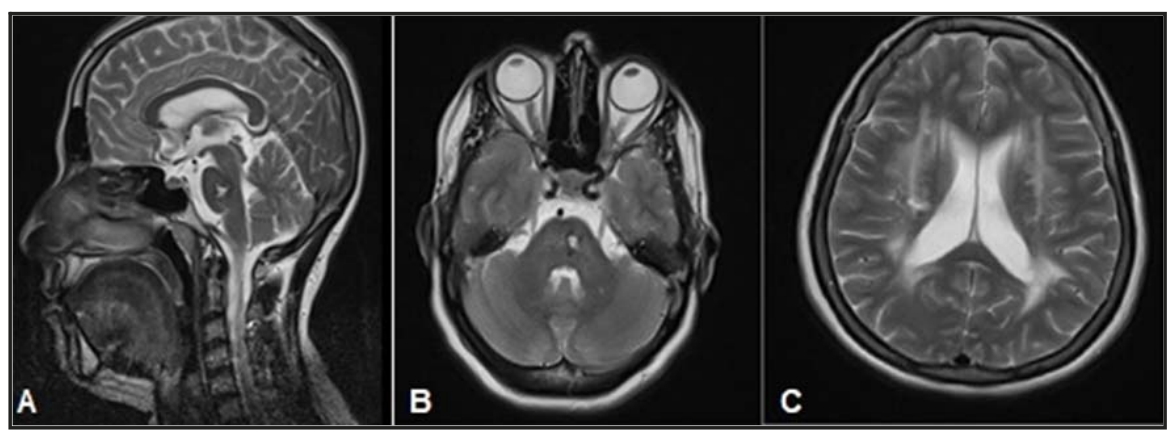

Figure 1: Brain MRI in a 35 year old patient with MS having clinical attacks, (A) Sagittal and (B, C) Axial T2-weighted image (3500/1 (06at the level of cerebellopontineangle and lateral ventricles, respectively, showing multiple oval shaped - hyper intense T2 lesions (arrows) seen at pons adjacent to the Lt. cerebellopontine peduncle and the deep with matter (periventricular region) on both sides of lateral ventricles. 
https:/ / doi.org/ 10.15218/ zjms.2021.006

Regarding gender, periventricular region was the commonest location affected and nearly with the same proportions for both males and females $(96.9 \%$ and $94.5 \%$, respectively), as shown in Table 3 . Twelve female patients (16.4\%) had basal ganglia lesions; such lesions were not found in males. Therefore, basal ganglia was the only statistically different site between males and females $(P=0.016)$. Otherwise, other lesions distribution showed no significant difference by gender. Regarding disease duration, the study revealed that new active lesions along with $\mathrm{Gd}$ contrast enhancement and restricted diffusion lesions were significantly associated with the duration of the disease $(P<0.001)$, shown in Table 4. On the other hand, there was no statistically significant difference between the old lesions (black holes) with disease duration $\left(P=0.146^{*}\right)$. A large tumefactive MS mass of size mean \pm SD of $26 \pm 3.6 \mathrm{~mm}$ was seen in only three cases that were acute on chronic cases (Figure 2), and there was no significant relation with disease duration. There was significant association between cortical lesions and disease duration $(P<0.001)$. One case showed normal appearing white matter, and it was a new case presented with neurological manifestations and a new MS lesion in another site rather than the brain.

Table 3: Distribution of the lesion in the brain MRI by gender $(\mathrm{N}=105)$.

\begin{tabular}{lccccc}
\hline $\begin{array}{l}\text { Variables of the lesion } \\
\text { site }\end{array}$ & \multicolumn{4}{c}{ Male (N=32) } & \multicolumn{2}{c}{ Female (N=73) } & $P$ value \\
& No. & $(\%)$ & No. & $(\%)$ & \\
\hline Periventricular region & 31 & $(96.9)$ & 69 & $(94.5)$ & $>0.999^{*}$ \\
Juxtacortical region & 22 & $(68.8)$ & 46 & $(63)$ & 0.571 \\
Corpus callosum & 10 & $(31.3)$ & 39 & $(53.4)$ & 0.036 \\
Cerebellum & 4 & $(12.5)$ & 16 & $(21.9)$ & 0.258 \\
Basal ganglia & 0 & $(0)$ & 12 & $(16.4)$ & $0.016^{*}$ \\
Mid brain & 1 & $(3.1)$ & 9 & $(12.3)$ & $0.277^{*}$ \\
Pons & 1 & $(3.1)$ & 12 & $(16.4)$ & $0.103^{*}$ \\
Medulla Oblongata & 1 & $(3.1)$ & 1 & $(1.4)$ & $0.519^{*}$ \\
\hline
\end{tabular}

*Indicate Fisher Exact Test

Table 4: Certain lesion characteristics and their association with duration of the disease.

\begin{tabular}{|c|c|c|c|c|c|c|c|}
\hline \multirow[t]{2}{*}{ Variable } & \multicolumn{2}{|c|}{$\begin{array}{l}\text { New cases } \\
(\mathrm{N}=11)\end{array}$} & \multicolumn{2}{|c|}{$\begin{array}{l}\text { Acute on chronic } \\
\qquad(\mathrm{N}=31)\end{array}$} & \multicolumn{2}{|c|}{$\begin{array}{l}\text { Chronic cases } \\
\qquad(\mathrm{N}=63)\end{array}$} & \multirow[t]{2}{*}{$P$ value } \\
\hline & No. & (\%) & No. & $(\%)$ & No. & (\%) & \\
\hline New active lesion & 4 & $(36.4)$ & 24 & $(77.5)$ & 0 & $(0)$ & $<0.001^{*}$ \\
\hline Gd enhancement & 4 & $(36.4)$ & 25 & $(80.6)$ & 0 & (0) & $<0.001$ \\
\hline DW positive images & 4 & $(36.4)$ & 23 & $(74.2)$ & 5 & (7.9) & $<0.001$ \\
\hline Old lesion/black hole & 0 & (0) & 7 & $(22.6)$ & 16 & $(25.4)$ & $0.146^{*}$ \\
\hline Tumetactive MS mass & 0 & (0) & 3 & $(9.7)$ & 0 & $(0)$ & $0.052^{*}$ \\
\hline Cortical lesions & 0 & (0) & 7 & $(22.6)$ & 0 & (0) & $<0.001^{*}$ \\
\hline Mild Brain atrophy & 0 & (0) & 3 & $(9.7)$ & 0 & (0) & 0.052 \\
\hline
\end{tabular}


In addition, the study showed a significant relation between DW and Gd. Enhancement $(P<0.001)$. However, five out of 32 patients $(15.6 \%)$ had restricted diffusion lesions and did not show Gd. enhancement, and vice versa, two patients out of $73(2.7 \%)$ had brain lesions showed Gd. Enhancement and not were restricted DW images (Table 5).

Table 5: Association between certain lesion characteristics.

\begin{tabular}{llcccc}
\hline & & $\begin{array}{c}\text { Gd. Enhancement } \\
\text { No. (\%) }\end{array}$ & $\begin{array}{c}\text { No enhancement } \\
\text { No. (\%) }\end{array}$ & $\begin{array}{c}\text { Total } \\
\text { No. (\%) }\end{array}$ & P value \\
\hline DW & Restricted & $27(84.4)$ & $5(15.6)$ & $32(100)$ & $<0.001$ \\
& Not restricted & $2(2.7)$ & $71(97.3)$ & $73(100)$ & \\
Total & 105 & $29(27.6)$ & $76(72.4)$ & $105(100)$ & \\
\hline
\end{tabular}

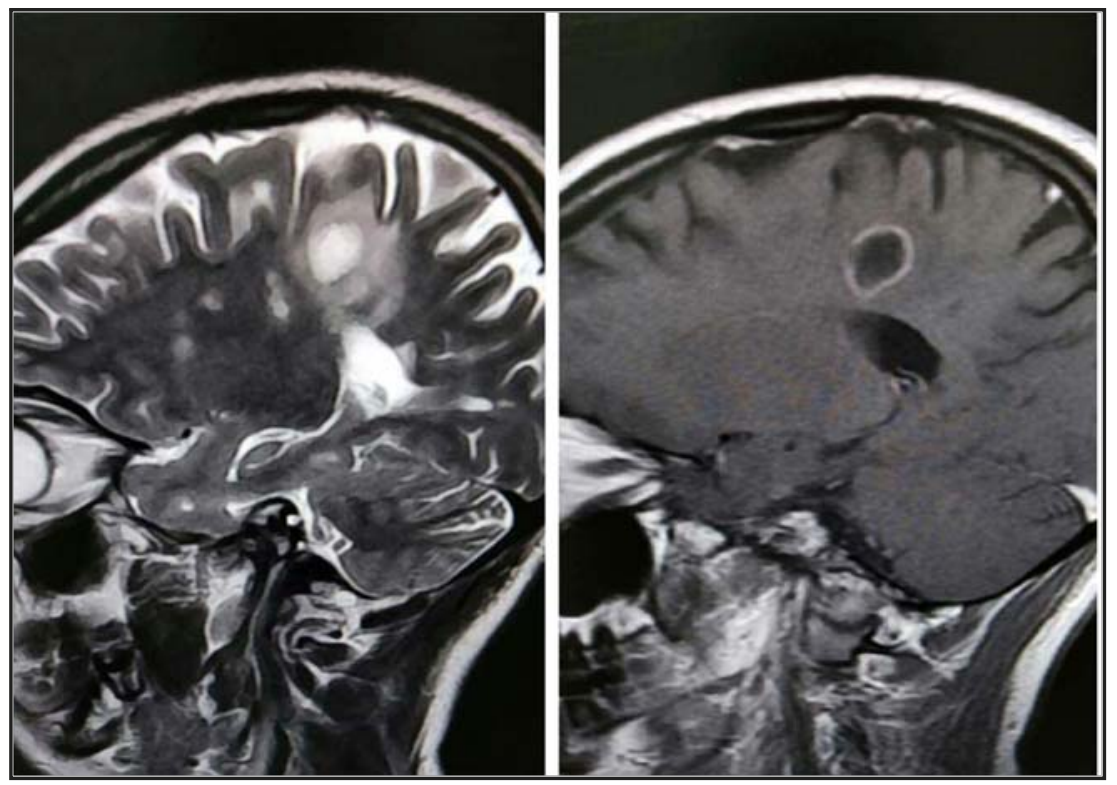

Figure 2: Brain MRI in a 45 year old patient with MS having a clinical exacerbation of symptoms, (A) Sagittal FLAIR (6000/90)and (B) contrast-enhanced T1-weighted image (550/17), showing multiple small hyperintense lesions with single hyperintense tumefactive oval mass (arrow) in the right frontoparietal region with extension to centrum semiovale, measuring more than $2 \mathrm{~cm}$ surrounded by edema and has incomplete ring enhancement. 


\section{Discussion}

There are extensive studies on MS in the literature dealing with the different aspects of the disease. It agreed that even when a patient is clinically consistent with MS, we still have to study the WMLs carefully to evaluate the distribution and assess the radiological manifestations of MS lesions. ${ }^{21}$ MS plaques can be characterized at MR imaging by their location, morphology, signal intensity, and degree of gadolinium enhancement. Many epidemiological studies went through different demographic aspects of the MS disease. According to a study done in the Multiple Sclerosis Clinic, Medical City in Baghdad, Iraq, the most common age group was 30-39 years with female to male ratio of $1.85: 1{ }^{14}$ The WHO published an Atlas review of the global MS epidemiology, in which they reported that neurological symptoms appear at around third decade of age and Iraq among the country of the age of onset 30-35 year. ${ }^{22}$ In the present study, the highest proportion of age group (36\%) was between $36-45$ year with females were nearly twice as males $(69.5 \%$ and $30.5 \%$, respectively), which were also compatible with other studies results that performed in Baghdad by Turkey et al. ${ }^{23}$ and in centers of MS of neighboring countries like Kuwait, Saudi Arabia, Jordan and Iran. ${ }^{24-26}$ Regarding lesion distribution in brain regions, the most common location was in the periventricular region, and it was found in more than $90 \%$ of MS patients. This result was in agreement with a study done by Ghassem et al., which revealed that adult MS patients were more likely to have supratentorial lesions (96.5\%). ${ }^{27}$ Another study done in Jordan and Iraq in 2008 by Al-Shimmery et al $^{28}$ reported that $98 \%$ of patients had the lesion in the deep white matter of the cerebrum. However, in the same study, $34.6 \%$ of patients had brain stem lesions, and $23.1 \%$ of the patients showed cerebellar lesions, which was higher than our measured percentage $(23.8 \%$ and $11.4 \%$, respectively). The present study revealed no statistically significant difference in lesion distribution by gender in all brain regions $(P>0.05)$ except for basal ganglia involvement, which was significantly different by gender. Basal ganglia lesions were seen in $16.4 \%$ of women and not seen in men $(P=0.016)$. Such a result was in agreement with a study done by Hasan et al. in 2011 on under 18 age MS patients, who also reported no significant difference in the distribution of the lesions between males and females. However, the basal ganglia lesions were also observed in females more than males. ${ }^{13}$ The present study demonstrated a topographic difference in characteristics lesion by MRI among new, chronic and chronic with active attacks. The highest proportion of patients having contrast enhanced lesions were either new $(36.4 \%)$ or acute on chronic $(80 \%)$. There was a statistically significant association between the presence of new enhancing lesions and the duration of the disease $(P<0.001)$. According to previous studies and the global recommended protocol, Gd injection was given to the patient to assess the activity and progress of the disease. $^{32,33}$ The leakage of intravenous gadolinium ( $\mathrm{Gd}$ ) into the parenchyma from a breakdown of blood brain barrier occurs early in the development of an MS lesion and serves as a useful marker of inflammation. ${ }^{34}$ Also, we observed that $36.4 \%$ of the new onset cases and about $75 \%$ of acute on chronic had restricted brain lesions on DWI, and there was a significant association $(P<0.001)$ with disease duration. Therefore, it is concluded that clinical exacerbation of symptoms is significantly associated with restricted diffusion lesions. Similarly, in most of the studies, the association between the presence of new lesions with restriction on DWI and exacerbation of neurological symptoms was statistically significant. ${ }^{17,35}$ On the other hand, nearly $25 \%$ of chronic cases' brains showed black hole lesions, while the new cases did not have black holes lesion in their brain MRI. The neural 
degeneration and destruction of neural axons results in the formation of irreversible $\mathrm{TI}$ hypointense lesions black holes, and it is more prominent and significant as disease progress and becomes more chronic. ${ }^{36}$ In this study, significantly, only the chronic cases with acute attacks had cortical lesions in their brain with a proportion of $22.6 \%$ $(P<0.001)$. This result was compatible with a study done by Choi et al. ${ }^{37}$ that revealed that cortical pathology and clinical exacerbation or progress of symptoms are secondary to leptomeningeal inflammation. About $10 \%$ of acute on chronic cases had tumefactive mass lesions. None of the other categories had this mass, which indicates that tumefactive lesions can only be expected in patients with clinical exacerbation of symptoms. The literature reported that tumefactive demyelination mass in MS is suspected when focal neurological signs present and appear abruptly within few days to weeks. ${ }^{38}$ However, there was no significant association between tumefactive mass and disease duration, which was also in agreement with a study done by Lucchinetti et al. that found that most tumefactive features did not correlate with gender, course, or diagnosis. ${ }^{39}$ Another no significant association was found between brain atrophy and disease duration $(P=0.052)$, and this was supported by several studies that concluded that brain atrophy was observed and continue regardless of disease course and MS subtype. ${ }^{40}$ Although optic neuritis is a common clinical manifestation in $\mathrm{MS}^{23}$ but optic nerve lesion was not analyzed in the present study sample. This is because the sensitivity of MRI alone for optic nerve involvement is low and needs to be correlated with clinical findings and few tests like optic coherence tomography, ${ }^{4}$ which were not included in the present study. Moreover, optic neuritis has a high recovery outcome post high dose steroids, and most patients were treated previously. ${ }^{41}$ Finally, our study showed a statistically significant relation between DW and Gd. Enhancement $(P<0.001)$, in which more than $90 \%$ of restricted diffusion brain lesions enhanced post Gd Contrast. Several studies revealed that the restriction diffusion of lesion and contrast enhancement were significantly correlated. ${ }^{27,35}$ However, hyper intense DWI finding does not necessarily overlap with contrast enhancement. ${ }^{35}$ Some studies revealed that $\mathrm{Gd}$ enhancement also does not necessitate DW restriction. ${ }^{17,42}$ Similarly, in the present study, less than $3 \%$ of cases with not restricted diffusion lesions but still showed enhancement post contrast. There are limitations of the present study, mainly due to the lack of High tesla MRI machines like $3 \mathrm{~T}$ in Erbil, which can give more accurate findings, and because the study was not correlated with clinical findings.

\section{Conclusion}

The study showed that MS lesions are distributed mainly in periventricular region, juxtacortical and corpus callosum, which was nearly of the same proportions observed by previous studies in the Middle East, precisely in countries like Iraq and Jordan. However, the proportions of the brain stem and cerebellum lesions appear to be lower than the above-mentioned studies. The proportions of MS lesions in basal ganglia were significantly higher in women. The lesion characteristics were significantly correlated with disease durations. Further studies with a larger sample of MS patients and fewer variables in this field are recommended to attain more accurate results. A comparison study of brain lesions with spinal cord lesions is advised for a comprehensive evaluation of MS lesions distribution in Erbil population, according to the last updated McDonald criteria 2017.

\section{Competing interests}

The authors declare no competing interests. 


\section{References}

1. Sarbu N, Shih RY, Jones RV, Horkayne-Szakaly I, Oleaga L, Smirniotopoulos JG. White matter diseases with radiologic-pathologic correlation. Radiographics 2016; 36(5):1426-47.

2. Filippi M, Rocca MA. MR imaging of multiple sclerosis. Radiology 2011; 259(3):659-81.

3. Rovira À, Wattjes MP, Tintoré M, Tur C, Yousry TA, Sormani MP, et al. Evidence-based guidelines: MAGNIMS consensus guidelines on the use of MRI in multiple sclerosis-clinical implementation in the diagnostic process. Nat Rev Neurol 2015; 11(8):471.

4. Thompson AJ, Banwell BL, Barkhof F, Carroll WM, Coetzee T, Comi G, et al. Diagnosis of multiple sclerosis: 2017 revisions of the McDonald criteria. Lancet Neurol 2018; 17(2):162-73.

5. Khamseh F, Rahimian E, Ommi Z, Abolhasani E, ShariatPanahi M. Fatigue in multiple sclerosis: cross-sectional correlation with brain MRI findings. Iran J Neurol 2010; 9(29):745-50.

6. Dutta R, Trapp BD. Relapsing and progressive forms of multiple sclerosis-insights from pathology. Current opinion in neurology. 2014; 27(3):271

7. Carroll WM. 2017 McDonald MS diagnostic criteria: Evidence-based revisions. Mult Scler J 2018; 24(2)92-5.

8. MoraalB, Wattjes MP, Geurts JJ, Knol DL, van Schijndel RA, Pouwels PJ, et al. Improved detection of active multiple sclerosis lesions: 3D subtraction imaging. Radiology 2010; 255(1):154 -63 .

9. Tardif CL, Bedell BJ, Eskildsen SF, Collins DL, Pike GB. Quantitative magnetic resonance imaging of cortical multiple sclerosis pathology. Mult Scler Int 2012; 2012:11.

10. Kira Jl. Multiple sclerosis in the Japanese population. Lancet Neurol2003;2(2):117-27.

11. Mohammed EM. Multiple sclerosis is prominent in the Gulf States. Pathogenesis 2016; 3(2):1938.

12. Al-Hamadani HA, Abdalla AS, Al-Saffar AJ. The course of early-onset multiple sclerosis in Iraqi children. World J Pediatr 2012; 8(1):47-51.

13. Hasan ZN, Hasan HA, Sabah AS. Clinical and radiological study of Iraqi multiple sclerosis patients with childhood onset. Neurosciences (Riyadh) 2011; 16(3):229-32.

14. Mohammed HA, Aboud HN, Hassan B. Multiple Sclerosis Clinic in Iraq, an Endeavourforan Unraveling Database. AJCEM 2018;6(3):69.

15. Falah Y, Al-Araji A. Multiple sclerosis in Iraq: History, epidemiology and the future. TOFIQ $J$ Med Sci 2014; 1(1):62-8.

16. Tatekawa $H$, Sakamoto $S$, Hori M, Kaichi $Y$, Kunimatsu A, Akazawa $\mathrm{K}$, et al. Imaging differences between neuromyelitisoptica spectrum disorders and multiple sclerosis: a multi-institutional study in Japan. AJRN 2018; 39(7):1239-47.
16. Brownlee WJ, Hardy TA, Fazekas F, Miller DH. Diagnosis of multiple sclerosis: progress and challenges. Lancet 2017; 389(10076):1336-46.

17. Altintas A, Petek B, Isik N, Terzi M, Bolukbasi F, Tavsanli $M$, et al. Clinical and radiological characteristics of tumefactive demyelinating lesions: follow-up study. Mult Scler J2012; 18(10):1448-53.

18. Sahraian MA, Radue EW, Haller S, Kappos L. Black holes in multiple sclerosis: definition, evolution, and clinical correlations. Acta Neurol Scand 2010; 122:1-8.

19. Stadelmann C, Wegner C, Brück W Inflammation, demyelination, and degenerationrecent insights from MS pathology. Biochimica et Biophysica Acta (BBA)-Molecular Basis of Disease 2011; 1812(2):275-82.

20. Charil A, Yousry TA, Rovaris M, Barkhof $F$, De Stefano N, Fazekas F, et al. MRI and the diagnosis of multiple sclerosis: expanding the concept of "no better explanation." Lancet Neurol 2006; 5(10):841-52.

21. World Health Organization. Atlas: multiple sclerosis resources in the world; 2008.

22. Turkey AM, Al-Fahid S, Al-Saffar K, Neurophthalmological manifestations in multiple sclerosis. Zanco J Med Sci 2010; 108(1):109-10.

23. Sahraian MA, Khorramnia S, Ebrahim MM, Moinfar Z, Lotfi J, Pakdaman H. Multiple sclerosis in Iran: a demographic study of 8,000 patients and changes over time. European Neurology 2010; 64(6):331-6.

24. Al-Araji A, Mohammed Al. Multiple sclerosis in Iraq: Does it have the same features encountered in Western countries? J Neurol Sci 2005; 234(1-2):67-71.

25. Alroughani R, Ahmed SF, Behbehani R, Khan R, Thussu A, Alexander KJ, et al. Increasing prevalence and incidence rates of multiple sclerosis in Kuwait. Mult Scler J 2014; 20(5):5437.

26. Davoudi Y, Foroughipour M, Torabi R, Layegh $\mathrm{P}$, Matin N, Shoeibi A. Diffusion weighted imaging in acute attacks of multiple sclerosis. Iran J Radiol 2016; 13(2).

27. El-Salem K, Al-Shimmery E, Horany K, Al-Refai A, Al-Hayk K, Khader Y. Multiple sclerosis in Jordan: a clinical and epidemiological study. J Neurol 2006; 253(9):1210-6.

28. Ghassemi R, Narayanan S, Banwell B, Sled JG, Shroff M, Arnold DL. Canadian Pediatric Demyelinating Disease Network. Quantitative determination of regional lesion volume and distribution in children and adults with relapsing-remitting multiple sclerosis. PLoS One 2014; 9(2):e85741.

29. Alroughani R, Al Hashel J, Lamdhade S, Ahmed SF. Predictors of conversion to multiple sclerosis in patients with clinical isolated syndrome using the 2010 revised McDonald criteria. ISRN Neurology 2012; 2012. 
30. Yamout B, Alroughani R, Al-Jumah M, Khoury $\mathrm{S}$, Abouzeid N, Dahdaleh M, et al. Consensus guidelines for the diagnosis and treatment of multiple sclerosis. Curr Med Res Opin 2013; 29(6):611-21.

31. Kira JI. Neuromyelitisoptica and opticospinal multiple sclerosis: mechanisms and pathogenesis. Pathophysiology 2011; 18(1):6979.

32. Al-Hashel J, Besterman AD, Wolfson C. The prevalence of multiple sclerosis in the Middle East. Neuroepidemiology 2008; 31(2):129-37.

33. Kasper DL, Braunwald E, Fauci AS, Hauser SL, Longo DL, Jameson JL, et al. Harrison's principles of internal medicine. $17^{\text {th }} \mathrm{ed}$. New York: McGraw-Hill Medical Publishing Division; 2008. P. 2611-20.

34. Lo CP, Kao HW, Chen SY, Chu CM, Hsu CC, Chen YC, et al. Comparison of diffusion-weighted imaging and contrastenhanced T1-weighted imaging on a single baseline MRI for demonstrating dissemination in time in multiple sclerosis. BMC Neurol 2014; 14(1):100.

35. Sahraian MA, Radue EW, Haller S, Kappos L. Black holes in multiple sclerosis: definition, evolution, and clinical correlations. Acta Neurol Scand 2010; 122(1):1-8.

36. Choi SR, Howell OW, Carassiti D, Magliozzi R, Gveric D, Muraro PA, et al. Meningeal inflammation plays a role in the pathology of primary progressive multiple sclerosis. Brain 2012; 135(10):2925-37.

37. Totaro R, Di Carmine C, Carolei A. Tumefactive demyelinating lesions in patients with relapsing remitting multiple sclerosis treated with Fingolimod. J Neurol Neurophysiol S 2014; 12:712.

38. Lucchinetti CF, Gavrilova RH, Metz I, Parisi JE, Scheithauer BW, Weigand S, et al. Clinical and radiographic spectrum of pathologically confirmed tumefactive multiple sclerosis. Brain 2008; 131(7):1759-75.

39. De Stefano N, Giorgio A, Battaglini M, Rovaris M, Sormani MP, Barkhof $F$, et al. Assessing brain atrophy rates in a large population of untreated multiple sclerosis subtypes. Neurology 2010; 74(23):1868-76.

40. Burton EV. Optic Neuritis: Clinical Manifestations, Pathophysiology, and Management. In Minagar A., editor. Neuroinflammation Academic Press; 2018. P. 337-53.

41. Chatterjee S, Commowick O, Afacan O, Warfield SK, Barillot C. Identification of Gadolinium contrast enhanced regions in MS lesions using brain tissue microstructure information obtained from diffusion and T2 relaxometry MRI. 21st International Conference on Medical Image Computing and Computer Assisted Intervention (MICCAI 2018), Sep 2018, Grenade, Spain. P. 63 -71 . 